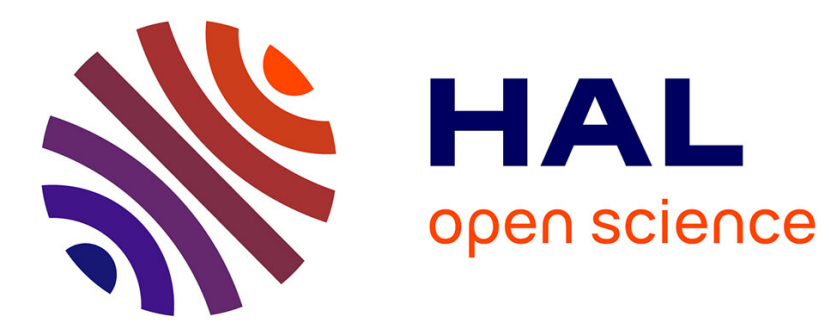

\title{
Sign and Magnitude of Spin Hamiltonian Parameters for Mn2+ Impurities in Calcite. A Multi- and Low-Frequency Study.
} Hogni Weihe, Stergios Piligkos

\section{- To cite this version:}

Hogni Weihe, Stergios Piligkos. Sign and Magnitude of Spin Hamiltonian Parameters for Mn2+ Impurities in Calcite. A Multi- and Low-Frequency Study.. Molecular Physics, 2008, 105 (15-16), pp.2025-2030. 10.1080/00268970701513856 . hal-00513121

\section{HAL Id: hal-00513121 https://hal.science/hal-00513121}

Submitted on 1 Sep 2010

HAL is a multi-disciplinary open access archive for the deposit and dissemination of scientific research documents, whether they are published or not. The documents may come from teaching and research institutions in France or abroad, or from public or private research centers.
L'archive ouverte pluridisciplinaire HAL, est destinée au dépôt et à la diffusion de documents scientifiques de niveau recherche, publiés ou non, émanant des établissements d'enseignement et de recherche français ou étrangers, des laboratoires publics ou privés. 


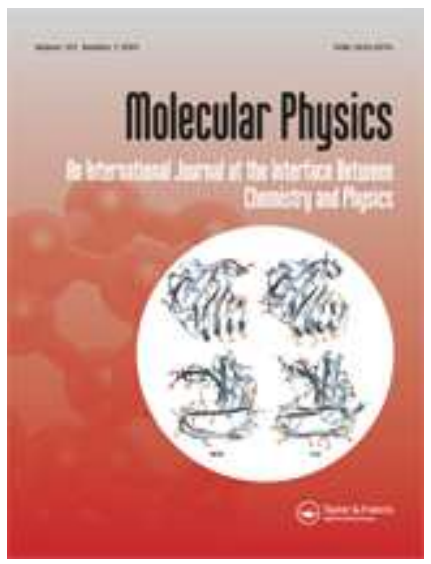

Sign and Magnitude of Spin Hamiltonian Parameters for Mn2+ Impurities in Calcite. A Multi- and Low-Frequency Study.

\begin{tabular}{|c|c|}
\hline Journal: & Molecular Physics \\
\hline Manuscript ID: & TMPH-2007-0108.R1 \\
\hline Manuscript Type: & Full Paper \\
\hline $\begin{array}{r}\text { Date Submitted by the } \\
\text { Author: }\end{array}$ & 30-May-2007 \\
\hline Complete List of Authors: & $\begin{array}{l}\text { Weihe, Hogni; University of Copenhagen, Dep. of Chemistry } \\
\text { Piligkos, Stergios; University of Copenhagen, Dep of Chemistry }\end{array}$ \\
\hline Keywords: & EPR spectroscopy, calcite, dolomite, marble, spin Hamiltonian \\
\hline \multicolumn{2}{|c|}{$\begin{array}{l}\text { Note: The following files were submitted by the author for peer review, but cannot be converted } \\
\text { to PDF. You must view these files (e.g. movies) online. }\end{array}$} \\
\hline calcite & \\
\hline
\end{tabular}

\section{S) ScholaroNE \\ Manuscript Central}




\title{
Sign and Magnitudes of Spin Hamiltonian
}

Parameters for $\mathrm{Mn}^{2+}$ Impurities in Calcite.

\section{A Multi- and Low-Frequency EPR Study.}

\author{
Stergios Piligkos*1, Ib Laursen ${ }^{2}$, Axel Morgenstjerne ${ }^{2}$ and \\ Høgni Weihe*1 \\ 1 Department of Chemistry, University of Copenhagen, Universitetsparken \\ 5, DK-2100 Copenhagen, Denmark \\ 2 Quantum Protein Centre, Technical University of Denmark, Building \\ 309, DK-2800 Lyngby, Denmark \\ * e-mails: weihe@kiku.dk, piligkos@kiku.dk
} June 14, 2007 


\begin{abstract}
L-, S-, and X-band powder EPR spectra and an L-band single-crystal spectrum of $\mathrm{Mn}^{2+}$ impurities in calcite are analysed by fitting of full spectral traces to a theoretical model. This analysis gives as parameters the traditional spin Hamiltonian parameters reflecting the three-fold symmetry of the cation lattice site as well as parameters determining the bandshape and width of the resonances. The three frequencies give a consistent description of the system. For this spin system it is shown that the relative sign of the axial zero-field splitting parameter $D$ and the hyperfine coupling constant $A$ may be safely determined at L-band.
\end{abstract}

\title{
Introduction
}

The EPR spectrum of $\mathrm{Mn}^{2+}$ ions present as an impurity in calcite, i.e. $\mathrm{CaCO}_{3}$ which crystallises in the space group $R \overline{3} c$ belonging to the trigonal crystal system, has for more than half a century [1] been a challenge for EPR spectroscopists. The $\mathrm{Mn}^{2+}$ ion in this lattice has a high-spin $3 \mathrm{~d}^{5}$ electronic configuration resulting in an orbitally non-degenerate $S=\frac{5}{2}$ ground state. The $\mathrm{Mn}^{2+}$ ion is coordinated to 6 oxide ligands from the carbonate anions resulting in a ligand field of $\mathrm{S}_{6}$ symmetry. In addition, the ${ }^{55} \mathrm{Mn}$ nucleus has a nuclear spin of $I=\frac{5}{2}$. This results in spectra exhibiting fine as well as hyperfine structure, resulting from the three-fold site symmetry and the electron-nucleus interaction, respectively. In the past, the parameters in the 
following spin Hamiltonian[2] eqn(1) have been determined.

$$
\begin{aligned}
\hat{H}= & \mu_{\mathrm{B}} g_{\perp}\left(\hat{S}_{x} B_{x}+\hat{S}_{y} B_{y}\right)+\mu_{\mathrm{B}} g_{z} \hat{S}_{z} B_{z}+A_{\perp}\left(\hat{S}_{x} \hat{I}_{x}+\hat{S}_{y} \hat{I}_{y}\right)+A_{\|} \hat{S}_{z} \hat{I}_{z} \\
& +D\left[\hat{S}_{z}^{2}-\frac{1}{3} S(S+1)\right]+B_{4}^{0} \hat{O}_{4}^{0}+B_{4}^{3} \hat{O}_{4}^{3} \\
& +P_{D}\left[\hat{I}_{z}^{2}-\frac{1}{3} I(I+1)\right]+P_{E}\left(\hat{I}_{x}^{2}-\hat{I}_{y}^{2}\right)-\mu_{\mathrm{N}} g_{\mathrm{N}}\left(\hat{I}_{x} B_{x}+\hat{I}_{y} B_{y}+\hat{I}_{z} B_{z}\right)
\end{aligned}
$$

where the first line accounts for the electronic Zeeman and the electron nucleus hyperfine interaction. The middle lines model the effect of the ligand field, and the bottom line accounts for the nuclear quadrupole term and the nuclear Zeeman interaction. Over the last 50 years, the following parameter values and ranges have been determined

$$
\begin{aligned}
& 87.0<\left|A_{\perp}\right| \approx\left|A_{\|}\right|<88.7 \\
& 74.9<|D|<76.4 \\
& 0.039<\left|B_{4}^{0}\right|<0.079 \\
& 0.34<\left|B_{4}^{3}\right|<17 \\
& 0.174<\left|P_{D}\right|<0.467 \\
& \left|P_{E}\right|=0.069 \\
& 1.997<g_{\perp}<2.0018 \\
& 2.001<g_{\|}<2.004 \\
& 2.00<g_{\mathrm{N}}<3.31
\end{aligned}
$$

where the parameters, except the $g$ factors, are all in units of $10^{-4} \mathrm{~cm}^{-1}$. Evidently, the numerical values of $A_{\perp}, A_{\|}, g_{\|}$and $g_{\perp}$ are found to lie within fairly narrow ranges. These values were, in most cases, extracted from the experimental spectra by using formulae from perturbation theory to various orders. Very recently, this subject was critically reviwed by ref[16], which introduced six (!) different methods to extract $D$ and $A$ values from the powder spectrum. 
To the best of our knowledge, the studies performed so far have all been performed at X-band frequencies, i.e. at 9-10 GHz, and at such relatively high frequencies the magnitude, not the sign, of the parameters $A=\left(A_{\perp} \approx A_{\|}\right)$, and $D$, by and large, determine the overall appearence of the spectrum; the remaining parameters being less important. The following three sign combinations of the hyperfine coupling constant $A$ and the axial zero-field splitting parameter $D$ have been reported in the earlier literature: $(D<0, A<0)[3,7,16],(D>0, A<0)[8,9,10,11,12]$, and $(D>0, A>0)$ $[1,5]$. Only two of the papers $[3,12]$ cited above seem to have acknowledged that only one sign combination of the values of $A$ and $D$ can possibly be correct. A sad detail is, however, that these authors disagree.

In this contribution we present experimental EPR spectra of powder samples of $\mathrm{Mn}^{2+}$ impurities in calcite recorded at 9.450, 3.866 and $1.1078 \mathrm{GHz}$, as well as a single-crystal spectrum obtained at $3.002 \mathrm{GHz}$. These spectra clearly allow us to, first, distinguish between the two possible relative-sign situations $A \times D>0$ or $A \times D<0$. Based on the fact that the nuclear $g_{\mathrm{N}}$ factor of ${ }^{55} \mathrm{Mn}$ is positive we are, finally, in the position of determining the absolute signs of $A$ and $D$.

\section{Results and Discussion}

An X-band powder spectrum of $\mathrm{CaCO}_{3}: \mathrm{Mn}^{2+}$ is shown in Figure 1. The X-band spectrum has been discussed in depth the last five decades, or so, and we will not repeat this discussion here. Every line and shoulder has been assigned to specific transitions within the 36 energy levels of this spin system. The correctness of these assignments depends, of course, on whether or not the right sign of $A$ is chosen. Of importance for this work, however, is 
the large variation of more than an order of magnitude in bandwiths of the numerous lines in the spectrum. The narrowest derivative-like lines have a peak-to-peak widths of less than $1 \mathrm{G}$ whereas some of the weaker absorptionlike lines have a full widths in half height in excess of $10 \mathrm{G}$.

Initially, the X-band spectrum was modelled with eqn (1) without the last three nuclear terms. This, already, resulted in good agreement yielding the residual designated as "b" in Figure 1. The very systematic appearence of this residual, especially at field values associated with the lines marked with an "f" in Figure 1 prompted for the nuclear terms to be present in the operator. These lines correspond to the 10 possible transitions within the $\pm \frac{1}{2}$ Kramers doublet having $\Delta M=1$ and $\Delta m= \pm 1, i$. e. the nuclear quantum number changes, and as a consequence these lines are sensitive to the nuclear parameters, i. e. the nuclear Zeeman effect and the quadrupole interaction. Inclusion of these terms in the Hamiltonian changed the fit from being just good to being absolutely excellent, see the residual designated as "c" in Figure 1, the computed spectrum is virtually identical with the experimental one.

The spin Hamiltonian parameters together with the bandwidth and bandshape parameters were extracted directly from the full spectrum by minimising the squared residuals as outlined in the Appendix. From the Xband spectrum we extracted the following spin Hamiltonian parameters: $g_{\perp}=2.00123(2), g_{\|}=2.00131(5), A_{\perp}=-88.23(1) \cdot 10^{-4} \mathrm{~cm}^{-1}, A_{\|}=$ $-87.60(4) \cdot 10^{-4} \mathrm{~cm}^{-1}, D=-76.0(2) \cdot 10^{-4} \mathrm{~cm}^{-1}, B_{4}^{0}=-0.047(3) \cdot 10^{-4} \mathrm{~cm}^{-1}$, $B_{4}^{3}=1.12(5) \cdot 10^{-4} \mathrm{~cm}^{-1}, g_{\mathrm{N}}=+1.38(5) \mathrm{cm}^{-1}, P_{D}=-0.176(12) \cdot 10^{-4} \mathrm{~cm}^{-1}$, $P_{E}=0.13(5) \cdot 10^{-4} \mathrm{~cm}^{-1}$. Here, the $\operatorname{digit}(\mathrm{s})$ in parentheses is the estimated standard deviation on the last digit(s) as given by the Levenberg-Marquard fitting routine [17]. Most of the parameter values agree well with the ab- 
solute values as found by previous investigators and reproduced in eqn(2). A noteworthy exception to this agreement is the nuclear $g_{\mathrm{N}}$ factor which is found outside the previously determined range, see eqs(2). The value found by us is, within one standard deviation, identical to that reported for the ${ }^{55} \mathrm{Mn}$ nucleus, namely $g_{\mathrm{N}}=+1.3844$.

In order to account for the bandwidth and bandshape the following parameters were introduced and determined: $w_{x}=w_{y}=0.89(2) \cdot 10^{-4} \mathrm{~cm}^{-1}$, $w_{z}=1.01(5) \cdot 10^{-4} \mathrm{~cm}^{-1}, \sigma_{D}=1.1(6) \cdot 10^{-4} \mathrm{~cm}^{-1}, \sigma_{E}=2.8(6) \cdot 10^{-4} \mathrm{~cm}^{-1}$, $a=0.87(5)$. The parameters $w_{x}, w_{y}$, and $w_{z}$ are the components of an anisotropic linewidth common for all energy level pairs, and the $a$ parameter describes the lorentzian/gaussian composition of the line shape, see the Appendix. The parameters $\sigma_{D}$ accounts for the fact that the $\mathrm{Mn}^{2+}$ centers are not all situated exactly in the same environment in the crystals. The result is that the spin Hamiltonian parameter $D$ has not a sharp value but is somehow distributed around a mean value; the mean value being the value reported above. We have assumed that this distribution is gaussian having a width of $\sigma_{D}$. It was necessary to also introduce a small spread in the rhombic spin Hamiltonian parameter $E$, i.e. the coefficient multiplying the spin operator term $\left(\hat{S}_{x}^{2}-\hat{S}_{y}^{2}\right)$. The mean value of the parameter $E$ is zero but due to crystal imperfections, the $\mathrm{Mn}^{2+}$ ions are not situated at sites having strict $\mathrm{S}_{6}$ symmetry. We note in passing, that a similar situation was found for $\Delta(+)\left[\mathrm{Cr}(\operatorname{chxn})_{3} \lambda \lambda \lambda\right]^{3+}$ doped into $\Delta(+)\left[\mathrm{Rh}(\operatorname{chxn})_{3} \lambda \lambda \lambda\right]\left(\mathrm{NO}_{3}\right)_{3} \cdot 3 \mathrm{H}_{2} \mathrm{O}(\operatorname{chxn}$ $=1,2$-diamino-cyclohexane) which also crystallises in a uniaxial space group with the cation ion on a three-fold axis [19]. We tried to introduce spreads in the other parameters of eqn (1); this did not improve the fits significantly, and therefore we have chosen $\sigma_{D}$ and $\sigma_{E}$ as the only bandwidth broadening parameters. 
The correctness of the parameter values, as extracted from the X-band powder spectrum, may now be verified by recording spectra at lower frequencies. Powder spectra at S and L band frequencies are shown in Figures 2 and 3 , respectively. The powder used in Figures 1, 2, and 3 was from the same calcite single crystal. Fitting these spectra to the same model as used above resulted in essentially the same parameter values with two exeptions. (1) The nuclear $g_{\mathrm{N}}$ factor becomes badly determined at low magnetic fields; this is expected since the energetic consequence of this term is small at lower fields. (2) The bandwidth parameters obtained from the S and L band spectra were higher, reflecting that these spectra were recorded with a slightly too large modulation amplitude. The computed spectra are also shown in Figures 2 and 3. The agreement is good. We tried to change the sign of one of the parameters $D$ or $A$. In the case of the $\mathrm{S}$ band spectrum, Figure 2, this gave a spectrum clearly different from that shown. In the case of the $\mathrm{L}$ band spectrum, Figure 3, this sign change resulted in a computed spectrum bearing no resemblence, at all, with the experimental spectrum. From the comparison of the experimental and computed L band spectra we can conclude that $D$ and $A$ have the same sign. The reason for this clear conclusion may be inferred from Figures 4 and 5 which shows the energy level diagram at low magnetic fields for two different sign combinations. At low magnetic fields the two situations result in qualitatively very different energy level diagrams. At $\mathrm{L}$ and $\mathrm{S}$ band, the microwave quantum is small compared to the splittings at zero magnetic field, see Figures 4 and 5. Therefore, the spectra depend dramatically on the sign combination, especially in the low-field end.

The acid test performed in order to verify our parameter values is shown in Figure 6, which shows a single-crystal spectrum recorded at $3 \mathrm{GHz}$ on a home-built instrument. See the caption of Figure 6 for relevant experimental 
details. The spectrum exhibits very uniform linewidths varying between 1.0 and 1.6 Gauss. This indicates a crystal of higher quality than that used for the powder spectra. Likewise, this indicates the use of a too large modulation amplitude which we had to choose in order to reduce the noise in, and the acquisition time of, the spectrum. At least 110 lines can be counted in the experimental spectrum. A computed spectrum obtained by using the above spin Hamiltonian parameters is shown as the lower trace in Figure 6. The line positions in the computed spectrum deviate on average by 0.4 Gauss, and for no line more than the bandwidth, from the the corresponding lines in the experimental spectrum. This agreement is acceptable taking into account that there is some uncertainty in the orientation of the crystal. The whole spectrum, and especially the number and placement of the very weak lowfield lines, is very sensitive to the relative signs of $A$ and $D$, as well as to the remaining spin Hamiltonian parameters.

In summary, we have demonstrated here that magnitudes and signs of the spin Hamiltonian parameters may be obtained from low-frequency EPR spectra at room temperature. The X-band spectrum indicates that the $D$ and $A$ parameters have the same sign. This was verified by the lower-frequency spectra. By making use of the fact that the nuclear $g_{\mathrm{N}}$ factor is positive, we conclude that the parameters $D$ and $A$ are both negative as only this sign combination of $g_{\mathrm{N}}, D$, and $A$ reproduces all spectra. Hence, we agree with reference [3], which discussed these matters based on temperature variations of low-temperature, presumably X-band, spectra, but without showing the experimental data.

Our discussion of the subject might be of importance for the field of archeometry which currently makes use of the $\mathrm{CaCO}_{3}: \mathrm{Mn}^{2+} \mathrm{X}$-band EPR spectrum as a probe for the provenance of marble blocks used in ancient 
sculptures and buildings around the Mediterranean bassin. One of the important parameters in this context is the calcite:dolomite ratio being characteristic for a specific location. This ratio cannot be obtained from the spectra unless the spectra are correctly modelled.

\section{Acknowledgements}

EPSRC EPR National service at the University of Manchester for use of the spectrometers. We are grateful to Ole Johnsen, National Geological Museum in Copenhagen, for providing us the high quality calcite crystal used for the $3 \mathrm{GHz}$ single-crystal experiment. 
Figure 1: Experimental and calculated X-band spectrum of $\mathrm{CaCO}_{3}: \mathrm{Mn}^{2+}$ are shown together as the traces desinated as "a". The experimental spectrum was obtained at room temperature with $\nu=9.450 \mathrm{GHz}$. The lower traces show the difference $y_{\text {obs }}-y_{\text {calc }}(\mathrm{b})$ without the terms with $P_{D}, P_{E}$, and $g_{N}$, and (c) with all the terms of eqn(1). The vertical scale is the same for all plots. The lines on each side of the "f"-s correspond to the transitions $\left|\frac{-1}{2}, m\right\rangle \rightarrow\left|\frac{1}{2}, m \pm 1\right\rangle$.

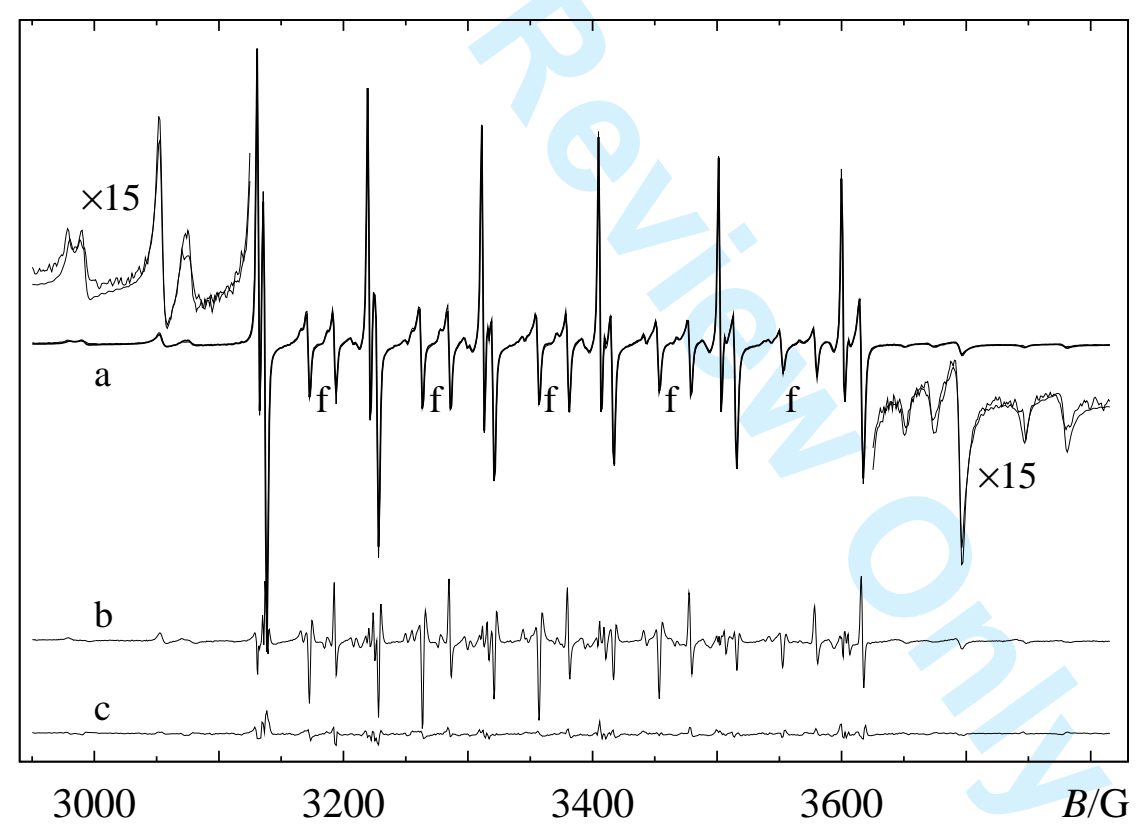


Figure 2: Experimental and calculated room-temperature S-band spectrum of $\mathrm{CaCO}_{3}: \mathrm{Mn}^{2+}$. The experimental spectrum (noisy trace) was obtained at room temperature with $\nu=3.8660 \mathrm{GHz}$.

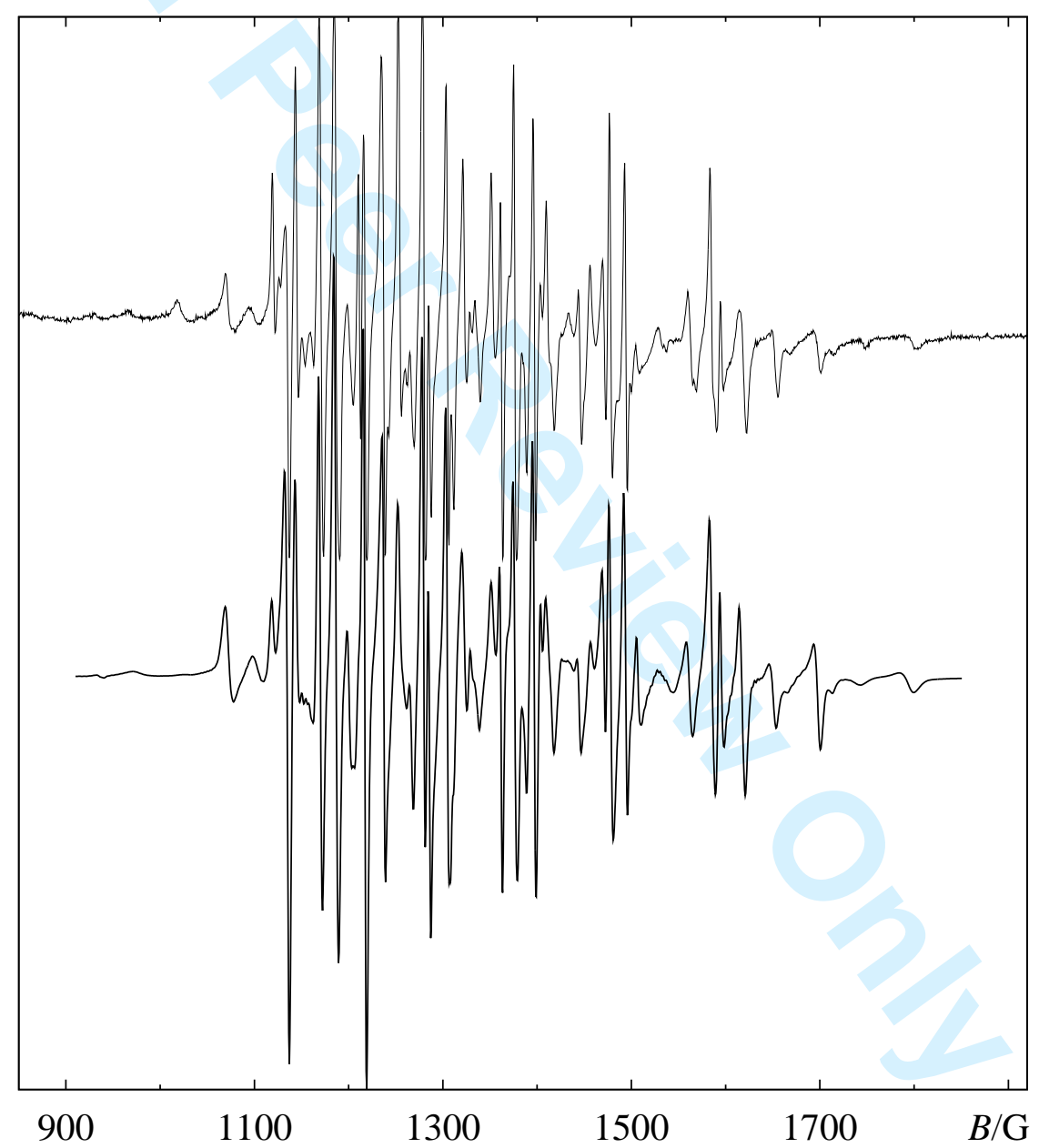


Figure 3: Experimental and calculated room-temperature L-band spectrum of $\mathrm{CaCO}_{3}: \mathrm{Mn}^{2+}$. The experimental spectrum (noisy trace) was obtained at room temperature with $\nu=1.1078 \mathrm{GHz}$.

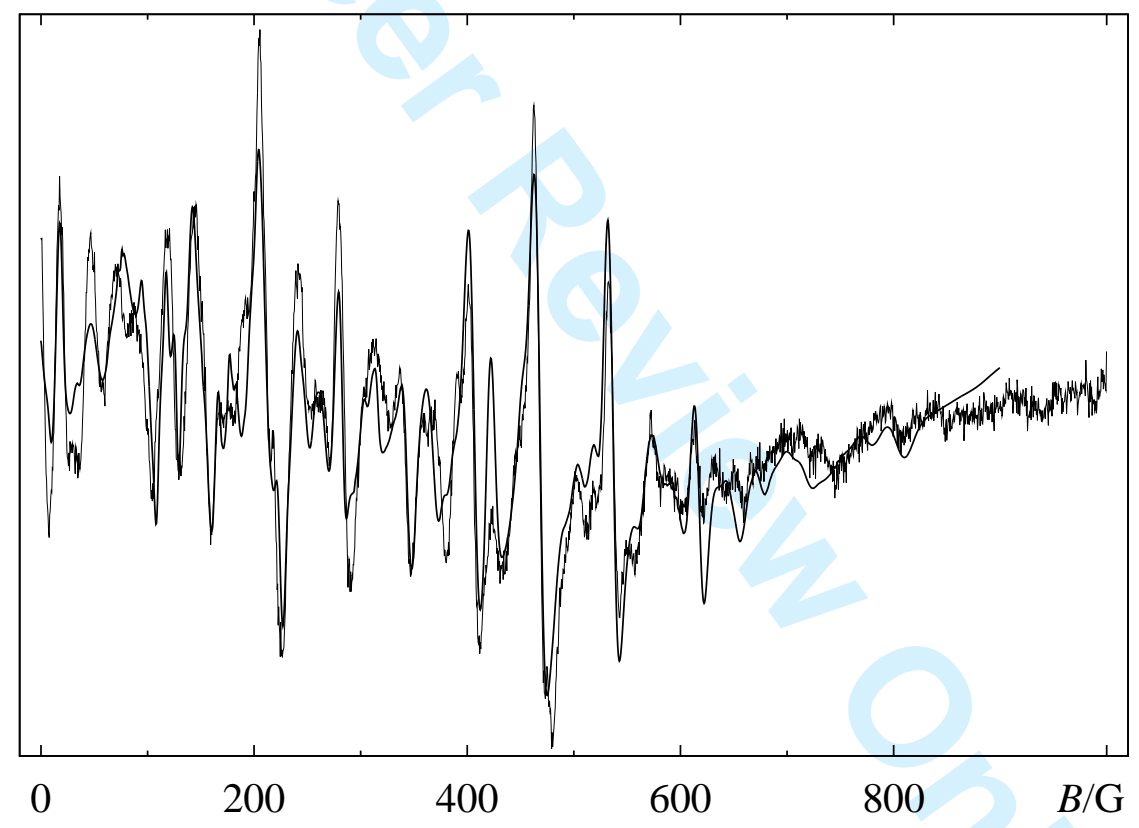


Figure 4: Energy level diagram obtained with $D<0, A_{\|}, A_{\perp}<0$, and $B \| z$. The arrow in the bottom of the figure indicates the energy equivalent of the microwave quantum $\nu=1.1078 \mathrm{GHz}$.

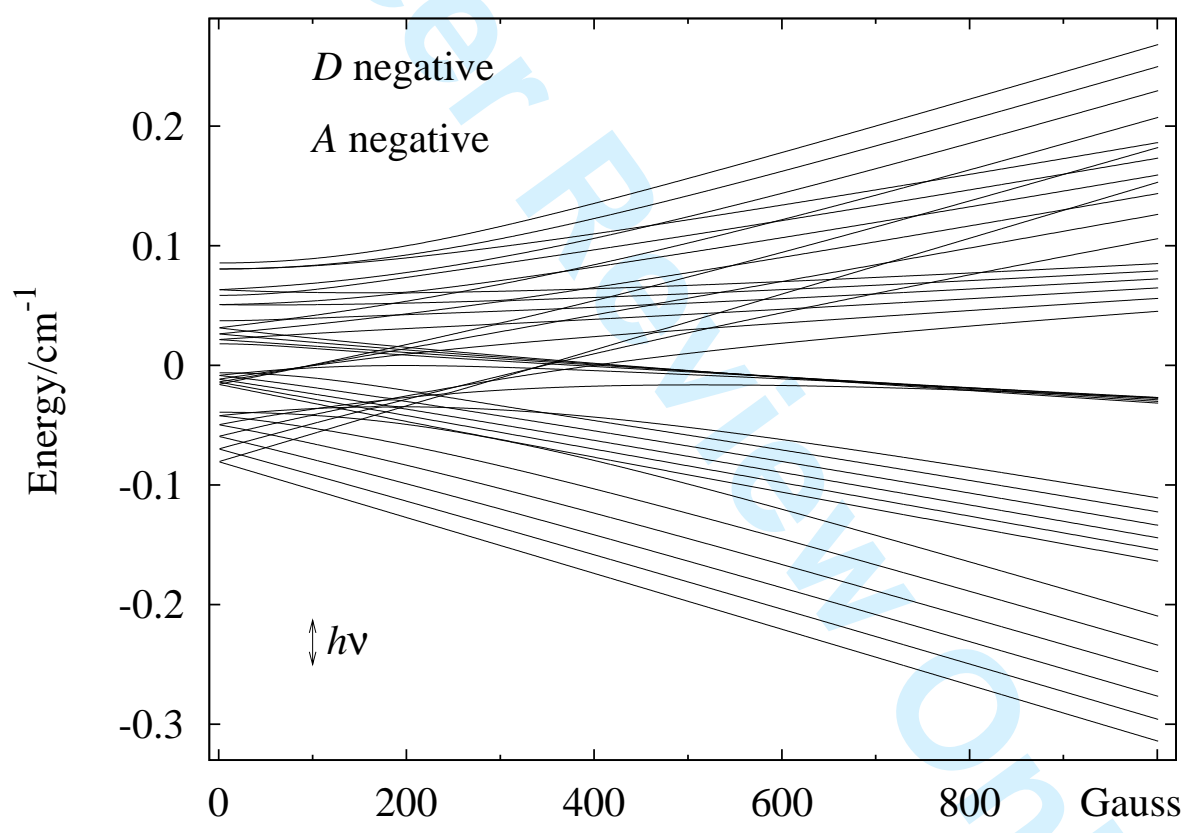


Figure 5: Energy level diagram obtained with $D<0, A_{\|}, A_{\perp}<0$, and $B \| z$. The arrow in the bottom of the figure indicates the energy equivalent of the microwave quantum for $\nu=1.1078 \mathrm{GHz}$.

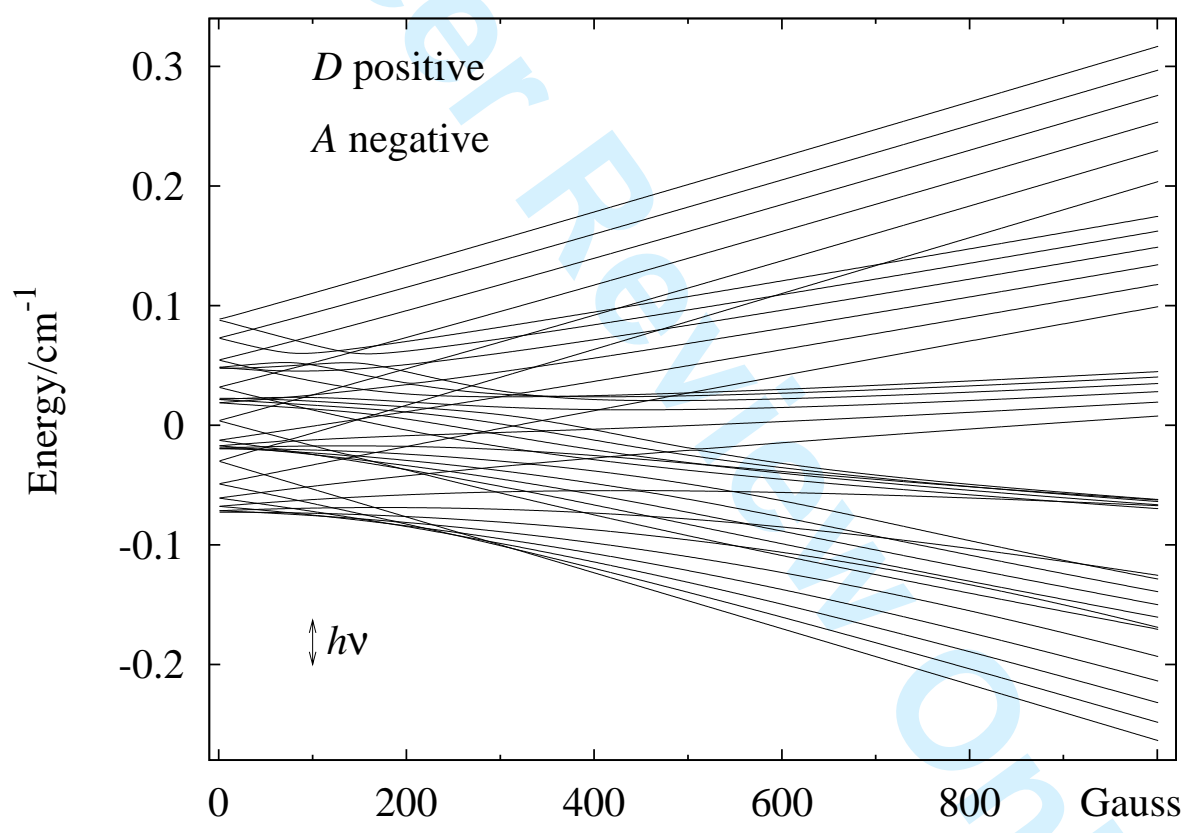


Figure 6: Single-crystal 3.002GHz room-temperature spectra of $\mathrm{Mn}^{2+}$ impurities in calcite in the field range 100-1750 Gauss. Upper trace: Experimental spectrum obtained by using a loop-gap resonator and the following experimental conditions: time constant 1 second, conversion time 3 seconds, modulation amplitude 1 Gauss, and a field resolution of 0.14 Gauss. No lines were detected above 1750 Gauss. Lower trace: Computed spectrum using the parameters given in the text. The angle between the unique crystallographic axis and the magnetic field was approximately 12 degrees.

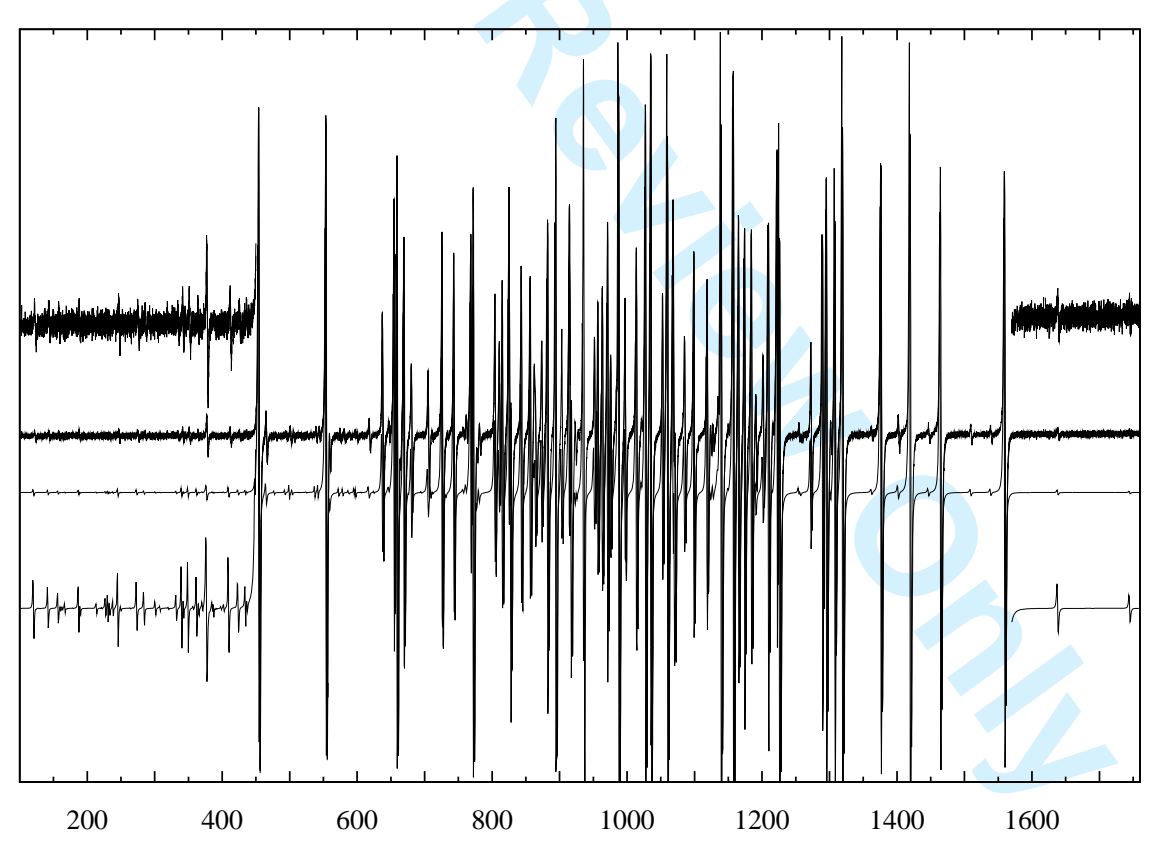




\section{A Computations}

The parameters of eqn (1) were extracted from the experimental spectra by standard $\chi^{2}$ minimization

$\chi^{2}=\sum_{i} \frac{\left[y_{\exp , i}\left(B_{i}\right)-y_{\text {calc }, i}\left(B_{i} ; \boldsymbol{q}\right)\right]^{2}}{\sigma_{i}^{2}}$

making use of the Leuvenberg-Marquardt algorithm [17]. $y_{\exp , i}$ and $y_{\text {calc }, i}$ is the experimental and calculated intensity, respectively, obtained for the $i$-th field value $B_{i} . \boldsymbol{q}$ designates the parameters to be extracted from the spectrum. $\sigma_{i}$ is the experimental uncertainty of the $i$-th experimental intensity. In our computations, this factor is composed of two terms

$\sigma_{i}^{2}=\sigma_{n}^{2}+\left(\frac{\partial y_{i}}{\partial B}\right)^{2} \sigma_{B}^{2}$

where $\sigma_{n}$ is the noise level common to all field points and $\sigma_{B}$ is the field uncertainty taken to be constant $(0.1 \mathrm{G})$ in the field interval.

At each field value the contribution to the derivative spectrum from any pair of energy levels $a, b$ is computed as the field derivative of

$y=\left(P_{a b} p_{a}-P_{b a} p_{b}\right) \times f\left(h \nu, \Delta_{a b}, \Gamma\right)$.

Here, $P_{a b}$ and $P_{b a}$ is the transition probability for the upward and downward transition, respectively. $p_{a}$ and $p_{b}$ is the thermal population of level $a$ and $b$, respectively. $f()$ is a bandshape function with the energy equivalent of the microwave radiation, the energy separation between levels $a$ and $b$, and a bandwidth as arguments, respectively.

In order to introduce some flexibility into the bandshape we use as bandshape function a popular approximation to the Voigt profile [20], namely a linear compination of a Lorentzian $L$ and a Gaussian $G$ lineshape with common bandwidth and centre

$f=a L+(1-a) G$ 
Finally, to account for the different bandwidth of the numerous lines in the spectra we model the bandwidth for the transition involving energy levels $a$ and $b$ as

$w_{a b}^{2}=w_{x}^{2} l_{x}^{2}+w_{y}^{2} l_{y}^{2}+w_{z}^{2} l_{z}^{2}+\sum_{i}\left(\frac{\partial \Delta_{a b}}{\partial q}\right)^{2} \sigma_{q_{i}}^{2}$

where $w_{x}, w_{y}$, and $w_{z}$ are common residual bandwidths for all energy level pairs, and $l_{x}, l_{y}$, and $l_{z}$ are direction cosines of the magnetic field vector with respect to the molecular coordinate system. The terms in the summation account for that the parameters do not have sharp values but rather may be distributed around a mean value. The form eqn (7) assumes that this parameter distribution is gaussian with a width of $\sigma_{q_{i}}$. We are well aware of that eqn (7) is only correct bandwidth to use if the bandshape itself as well as parameter distribution are both gaussian. For a bandshape described by eqn (6), eqn (7) should be modified. However, such a modification would not alter the qualitative outcome of using eqn (7), namely that transitions involving energy levels being more or less sensitive to the parameter $q_{i}$ will become broad and narrow, respectively. 


\section{References}

[1] Hurd, F. K.; Sachs, M.; Hershberger, W. D. Phys. Rev. 1954, 93, 373383

[2] Abragam, A.; Bleaney, B. Electron Paramagnetic Resonance of Transition Ions. Oxford University Press, Oxford 1970

[3] Matarese, L. M. J. Chem. Phys. 1961, 34, 336. Kikuchi, C.; Matarese, L. M. J. Chem. Phys. 1960, 33, 601-606

[4] Hodges, J.A.; Serway, R. A. J. Chem. Phys. 1968, 49, 2857-2858

[5] Mankowitz, J.; Low W. Phys. Rev. 1970, 2, 28-32

[6] Golding, R. M.; Tennant, W. C. Mol. Phys. 1974, 28, 167-175

[7] Tennant, W. C. J. Magn. Reson. 1974, 14, 152-159

[8] Barberis, G. E; Calvo, R.; Maldonado, H.G. Zarate, C.E. Phys. Rev. B $1975,12,853-860$

[9] Eidels-Dubovoi, S.; Beltrán-Lopez, V. J. Magn. Reson. 1978, 32, 441449

[10] Beltrán-Lopez, V.; Castro-Tello, J. J. Magn. Reson. 1980, 39, 437-460

[11] Beltrán-Lopez, V.; Jiménez, J. J. Magn. Reson. 1982, 48, 302-308

[12] Shepherd, R. A.; Graham, W. R. M. J. Chem. Phys. 1984, 81, 60806084

[13] Armiento, G.; Attanasio, D.; Platania, R. Archaeometry 1997, 39, 309319 
[14] Duliu, O. G.; Dinescu, L. C.; Skliros, D. J. Trace Microprobe Tech. 1999, 17, 165-175

[15] Attanasio, D.; Platania, R. J. Magn. Reson. 2000, 144, 322-329

[16] Garribba, E.; Micera, Giovanni. Magn. Res. Chem. 2006, 44, 11-19

[17] Preuss, W. H.; Flannery, B. P.; Teukolsky, S. A.; Vetterling, W. T. Numerical Recipes in $C$ : The art of scientific computing Cambridge University Press 1988

[18] Mabbs, F E.; Collison, D. Electron Paramagnetic Resonance of d Transition Metal Compounds, Elsevier 1992

[19] Klitgaard, S. K.; Galsbøl, F; Weihe, H. Spectrochimica Acta 2006, A63, $836-839$

[20] Bruce, S. D.; Higinbotham, J.; Marshall, I.; Beswich, P. H. J. Magn. Reson. 2000, 142, 57-63 


\title{
Sign and Magnitudes of Spin Hamiltonian \\ Parameters for $\mathrm{Mn}^{2+}$ Impurities in Calcite. A Multi- and Low-Frequency EPR Study.
}

\author{
Stergios Piligkos*1, Ib Laursen ${ }^{2}$, Axel Morgenstjerne ${ }^{2}$ and \\ Høgni Weihe*1 \\ 1 Department of Chemistry, University of Copenhagen, Universitetsparken \\ 5, DK-2100 Copenhagen, Denmark \\ 2 Quantum Protein Centre, Technical University of Denmark, Building \\ 309, DK-2800 Lyngby, Denmark \\ * e-mails: weihe@kiku.dk, piligkos@kiku.dk \\ June 14, 2007
}




\begin{abstract}
L-, S-, and X-band powder EPR spectra and an L-band single-crystal spectrum of $\mathrm{Mn}^{2+}$ impurities in calcite are analysed by fitting of full spectral traces to a theoretical model. This analysis gives as parameters the traditional spin Hamiltonian parameters reflecting the three-fold symmetry of the cation lattice site as well as parameters determining the bandshape and width of the resonances. The three frequencies give a consistent description of the system. For this spin system it is shown that the relative sign of the axial zero-field splitting parameter $D$ and the hyperfine coupling constant $A$ may be safely determined at L-band.
\end{abstract}

\title{
Introduction
}

The EPR spectrum of $\mathrm{Mn}^{2+}$ ions present as an impurity in calcite, i.e. $\mathrm{CaCO}_{3}$ which crystallises in the space group $\mathrm{R} \overline{3} \mathrm{c}$ belonging to the trigonal crystal system, has for more than half a century [1] been a challenge for EPR spectroscopists. The $\mathrm{Mn}^{2+}$ ion in this lattice has a high-spin $3 \mathrm{~d}^{5}$ electronic configuration resulting in an orbitally non-degenerate $S=\frac{5}{2}$ ground state. The $\mathrm{Mn}^{2+}$ ion is coordinated to 6 oxide ligands from the carbonate anions resulting in a ligand field of $\mathrm{S}_{6}$ symmetry. In addition, the ${ }^{55} \mathrm{Mn}$ nucleus has a nuclear spin of $I=\frac{5}{2}$. This results in spectra exhibiting fine as well as hyperfine structure, resulting from the three-fold site symmetry and the electron-nucleus interaction, respectively. In the past, the parameters in the 
following spin Hamiltonian[2] eqn(1) have been determined.

$$
\begin{aligned}
\hat{H}= & \mu_{\mathrm{B}} g_{\perp}\left(\hat{S}_{x} B_{x}+\hat{S}_{y} B_{y}\right)+\mu_{\mathrm{B}} g_{z} \hat{S}_{z} B_{z}+A_{\perp}\left(\hat{S}_{x} \hat{I}_{x}+\hat{S}_{y} \hat{I}_{y}\right)+A_{\|} \hat{S}_{z} \hat{I}_{z} \\
& +D\left[\hat{S}_{z}^{2}-\frac{1}{3} S(S+1)\right]+B_{4}^{0} \hat{O}_{4}^{0}+B_{4}^{3} \hat{O}_{4}^{3} \\
& +P_{D}\left[\hat{I}_{z}^{2}-\frac{1}{3} I(I+1)\right]+P_{E}\left(\hat{I}_{x}^{2}-\hat{I}_{y}^{2}\right)-\mu_{\mathrm{N}} g_{\mathrm{N}}\left(\hat{I}_{x} B_{x}+\hat{I}_{y} B_{y}+\hat{I}_{z} B_{z}\right)
\end{aligned}
$$

where the first line accounts for the electronic Zeeman and the electron nucleus hyperfine interaction. The middle lines model the effect of the ligand field, and the bottom line accounts for the nuclear quadrupole term and the nuclear Zeeman interaction. Over the last 50 years, the following parameter values and ranges have been determined

$$
\begin{aligned}
& 87.0<\left|A_{\perp}\right| \approx\left|A_{\|}\right|<88.7 \\
& 74.9<|D|<76.4 \\
& 0.039<\left|B_{4}^{0}\right|<0.079 \\
& 0.34<\left|B_{4}^{3}\right|<17 \\
& 0.174<\left|P_{D}\right|<0.467 \\
& \left|P_{E}\right|=0.069 \\
& 1.997<g_{\perp}<2.0018 \\
& 2.001<g_{\|}<2.004 \\
& 2.00<g_{\mathrm{N}}<3.31
\end{aligned}
$$

where the parameters, except the $g$ factors, are all in units of $10^{-4} \mathrm{~cm}^{-1}$. Evidently, the numerical values of $A_{\perp}, A_{\|}, g_{\|}$and $g_{\perp}$ are found to lie within fairly narrow ranges. These values were, in most cases, extracted from the experimental spectra by using formulae from perturbation theory to various orders. Very recently, this subject was critically reviwed by ref[16], which introduced six (!) different methods to extract $D$ and $A$ values from the powder spectrum. 
To the best of our knowledge, the studies performed so far have all been performed at X-band frequencies, i.e. at 9-10 GHz, and at such relatively high frequencies the magnitude, not the sign, of the parameters $A=\left(A_{\perp} \approx A_{\|}\right)$, and $D$, by and large, determine the overall appearence of the spectrum; the remaining parameters being less important. The following three sign combinations of the hyperfine coupling constant $A$ and the axial zero-field splitting parameter $D$ have been reported in the earlier literature: $(D<0, A<0)[3,7,16],(D>0, A<0)[8,9,10,11,12]$, and $(D>0, A>0)$ $[1,5]$. Only two of the papers[3, 12] cited above seem to have acknowledged that only one sign combination of the values of $A$ and $D$ can possibly be correct. A sad detail is, however, that these authors disagree.

In this contribution we present experimental EPR spectra of powder samples of $\mathrm{Mn}^{2+}$ impurities in calcite recorded at 9.450, 3.866 and $1.1078 \mathrm{GHz}$, as well as a single-crystal spectrum obtained at $3.002 \mathrm{GHz}$. These spectra clearly allow us to, first, distinguish between the two possible relative-sign situations $A \times D>0$ or $A \times D<0$. Based on the fact that the nuclear $g_{\mathrm{N}}$ factor of ${ }^{55} \mathrm{Mn}$ is positive we are, finally, in the position of determining the absolute signs of $A$ and $D$.

\section{Results and Discussion}

An X-band powder spectrum of $\mathrm{CaCO}_{3}: \mathrm{Mn}^{2+}$ is shown in Figure 1. The X-band spectrum has been discussed in depth the last five decades, or so, and we will not repeat this discussion here. Every line and shoulder has been assigned to specific transitions within the 36 energy levels of this spin system. The correctness of these assignments depends, of course, on whether or not the right sign of $A$ is chosen. Of importance for this work, however, is 
the large variation of more than an order of magnitude in bandwiths of the numerous lines in the spectrum. The narrowest derivative-like lines have a peak-to-peak widths of less than $1 \mathrm{G}$ whereas some of the weaker absorptionlike lines have a full widths in half height in excess of $10 \mathrm{G}$.

Initially, the X-band spectrum was modelled with eqn (1) without the last three nuclear terms. This, already, resulted in good agreement yielding the residual designated as " $b$ " in Figure 1. The very systematic appearence of this residual, especially at field values associated with the lines marked with an "f" in Figure 1 prompted for the nuclear terms to be present in the operator. These lines correspond to the 10 possible transitions within the $\pm \frac{1}{2}$ Kramers doublet having $\Delta M=1$ and $\Delta m= \pm 1, i$. e. the nuclear quantum number changes, and as a consequence these lines are sensitive to the nuclear parameters, i. e. the nuclear Zeeman effect and the quadrupole interaction. Inclusion of these terms in the Hamiltonian changed the fit from being just good to being absolutely excellent, see the residual designated as "c" in Figure 1, the computed spectrum is virtually identical with the experimental one.

The spin Hamiltonian parameters together with the bandwidth and bandshape parameters were extracted directly from the full spectrum by minimising the squared residuals as outlined in the Appendix. From the $\mathrm{X}$ band spectrum we extracted the following spin Hamiltonian parameters: $g_{\perp}=2.00123(2), g_{\|}=2.00131(5), A_{\perp}=-88.23(1) \cdot 10^{-4} \mathrm{~cm}^{-1}, A_{\|}=$ $-87.60(4) \cdot 10^{-4} \mathrm{~cm}^{-1}, D=-76.0(2) \cdot 10^{-4} \mathrm{~cm}^{-1}, B_{4}^{0}=-0.047(3) \cdot 10^{-4} \mathrm{~cm}^{-1}$, $B_{4}^{3}=1.12(5) \cdot 10^{-4} \mathrm{~cm}^{-1}, g_{\mathrm{N}}=+1.38(5) \mathrm{cm}^{-1}, P_{D}=-0.176(12) \cdot 10^{-4} \mathrm{~cm}^{-1}$, $P_{E}=0.13(5) \cdot 10^{-4} \mathrm{~cm}^{-1}$. Here, the $\operatorname{digit}(\mathrm{s})$ in parentheses is the estimated standard deviation on the last $\operatorname{digit}(\mathrm{s})$ as given by the Levenberg-Marquard fitting routine [17]. Most of the parameter values agree well with the ab- 
solute values as found by previous investigators and reproduced in eqn(2). A noteworthy exception to this agreement is the nuclear $g_{\mathrm{N}}$ factor which is found outside the previously determined range, see eqs $(2)$. The value found by us is, within one standard deviation, identical to that reported for the ${ }^{55} \mathrm{Mn}$ nucleus, namely $g_{\mathrm{N}}=+1.3844$.

In order to account for the bandwidth and bandshape the following parameters were introduced and determined: $w_{x}=w_{y}=0.89(2) \cdot 10^{-4} \mathrm{~cm}^{-1}$, $w_{z}=1.01(5) \cdot 10^{-4} \mathrm{~cm}^{-1}, \sigma_{D}=1.1(6) \cdot 10^{-4} \mathrm{~cm}^{-1}, \sigma_{E}=2.8(6) \cdot 10^{-4} \mathrm{~cm}^{-1}$, $a=0.87(5)$. The parameters $w_{x}, w_{y}$, and $w_{z}$ are the components of an anisotropic linewidth common for all energy level pairs, and the $a$ parameter describes the lorentzian/gaussian composition of the line shape, see the Appendix. The parameters $\sigma_{D}$ accounts for the fact that the $\mathrm{Mn}^{2+}$ centers are not all situated exactly in the same environment in the crystals. The result is that the spin Hamiltonian parameter $D$ has not a sharp value but is somehow distributed around a mean value; the mean value being the value reported above. We have assumed that this distribution is gaussian having a width of $\sigma_{D}$. It was necessary to also introduce a small spread in the rhombic spin Hamiltonian parameter $E$, i.e. the coefficient multiplying the spin operator term $\left(\hat{S}_{x}^{2}-\hat{S}_{y}^{2}\right)$. The mean value of the parameter $E$ is zero but due to crystal imperfections, the $\mathrm{Mn}^{2+}$ ions are not situated at sites having strict $\mathrm{S}_{6}$ symmetry. We note in passing, that a similar situation was found for $\Delta(+)\left[\operatorname{Cr}(\operatorname{chxn})_{3} \lambda \lambda \lambda\right]^{3+}$ doped into $\Delta(+)\left[\operatorname{Rh}(\operatorname{chxn})_{3} \lambda \lambda \lambda\right]\left(\mathrm{NO}_{3}\right)_{3} \cdot 3 \mathrm{H}_{2} \mathrm{O}(\operatorname{chxn}$ $=1,2$-diamino-cyclohexane) which also crystallises in a uniaxial space group with the cation ion on a three-fold axis [19]. We tried to introduce spreads in the other parameters of eqn (1); this did not improve the fits significantly, and therefore we have chosen $\sigma_{D}$ and $\sigma_{E}$ as the only bandwidth broadening parameters. 
The correctness of the parameter values, as extracted from the X-band powder spectrum, may now be verified by recording spectra at lower frequencies. Powder spectra at S and L band frequencies are shown in Figures 2 and 3, respectively. The powder used in Figures 1, 2, and 3 was from the same calcite single crystal. Fitting these spectra to the same model as used above resulted in essentially the same parameter values with two exeptions. (1) The nuclear $g_{\mathrm{N}}$ factor becomes badly determined at low magnetic fields; this is expected since the energetic consequence of this term is small at lower fields. (2) The bandwidth parameters obtained from the S and L band spectra were higher, reflecting that these spectra were recorded with a slightly too large modulation amplitude. The computed spectra are also shown in Figures 2 and 3. The agreement is good. We tried to change the sign of one of the parameters $D$ or $A$. In the case of the $\mathrm{S}$ band spectrum, Figure 2, this gave a spectrum clearly different from that shown. In the case of the L band spectrum, Figure 3, this sign change resulted in a computed spectrum bearing no resemblence, at all, with the experimental spectrum. From the comparison of the experimental and computed L band spectra we can conclude that $D$ and $A$ have the same sign. The reason for this clear conclusion may be inferred from Figures 4 and 5 which shows the energy level diagram at low magnetic fields for two different sign combinations. At low magnetic fields the two situations result in qualitatively very different energy level diagrams. At L and $\mathrm{S}$ band, the microwave quantum is small compared to the splittings at zero magnetic field, see Figures 4 and 5. Therefore, the spectra depend dramatically on the sign combination, especially in the low-field end.

The acid test performed in order to verify our parameter values is shown in Figure 6, which shows a single-crystal spectrum recorded at $3 \mathrm{GHz}$ on a home-built instrument. See the caption of Figure 6 for relevant experimental 
details. The spectrum exhibits very uniform linewidths varying between 1.0 and 1.6 Gauss. This indicates a crystal of higher quality than that used for the powder spectra. Likewise, this indicates the use of a too large modulation amplitude which we had to choose in order to reduce the noise in, and the acquisition time of, the spectrum. At least 110 lines can be counted in the experimental spectrum. A computed spectrum obtained by using the above spin Hamiltonian parameters is shown as the lower trace in Figure 6. The line positions in the computed spectrum deviate on average by 0.4 Gauss, and for no line more than the bandwidth, from the the corresponding lines in the experimental spectrum. This agreement is acceptable taking into account that there is some uncertainty in the orientation of the crystal. The whole spectrum, and especially the number and placement of the very weak lowfield lines, is very sensitive to the relative signs of $A$ and $D$, as well as to the remaining spin Hamiltonian parameters.

In summary, we have demonstrated here that magnitudes and signs of the spin Hamiltonian parameters may be obtained from low-frequency EPR spectra at room temperature. The X-band spectrum indicates that the $D$ and $A$ parameters have the same sign. This was verified by the lower-frequency spectra. By making use of the fact that the nuclear $g_{\mathrm{N}}$ factor is positive, we conclude that the parameters $D$ and $A$ are both negative as only this sign combination of $g_{\mathrm{N}}, D$, and $A$ reproduces all spectra. Hence, we agree with reference [3], which discussed these matters based on temperature variations of low-temperature, presumably X-band, spectra, but without showing the experimental data.

Our discussion of the subject might be of importance for the field of archeometry which currently makes use of the $\mathrm{CaCO}_{3}: \mathrm{Mn}^{2+} \mathrm{X}$-band $\mathrm{EPR}$ spectrum as a probe for the provenance of marble blocks used in ancient 
sculptures and buildings around the Mediterranean bassin. One of the important parameters in this context is the calcite:dolomite ratio being characteristic for a specific location. This ratio cannot be obtained from the spectra unless the spectra are correctly modelled.

\section{Acknowledgements}

EPSRC EPR National service at the University of Manchester for use of the spectrometers. We are grateful to Ole Johnsen, National Geological Museum in Copenhagen, for providing us the high quality calcite crystal used for the $3 \mathrm{GHz}$ single-crystal experiment. 
Figure 1: Experimental and calculated X-band spectrum of $\mathrm{CaCO}_{3}: \mathrm{Mn}^{2+}$ are shown together as the traces desinated as "a". The experimental spectrum was obtained at room temperature with $\nu=9.450 \mathrm{GHz}$. The lower traces show the difference $y_{\text {obs }}-y_{\text {calc }}\left(\right.$ b) without the terms with $P_{D}, P_{E}$, and $g_{N}$, and (c) with all the terms of eqn(1). The vertical scale is the same for all plots. The lines on each side of the "f"-s correspond to the transitions $\left|\frac{-1}{2}, m\right\rangle \rightarrow\left|\frac{1}{2}, m \pm 1\right\rangle$.

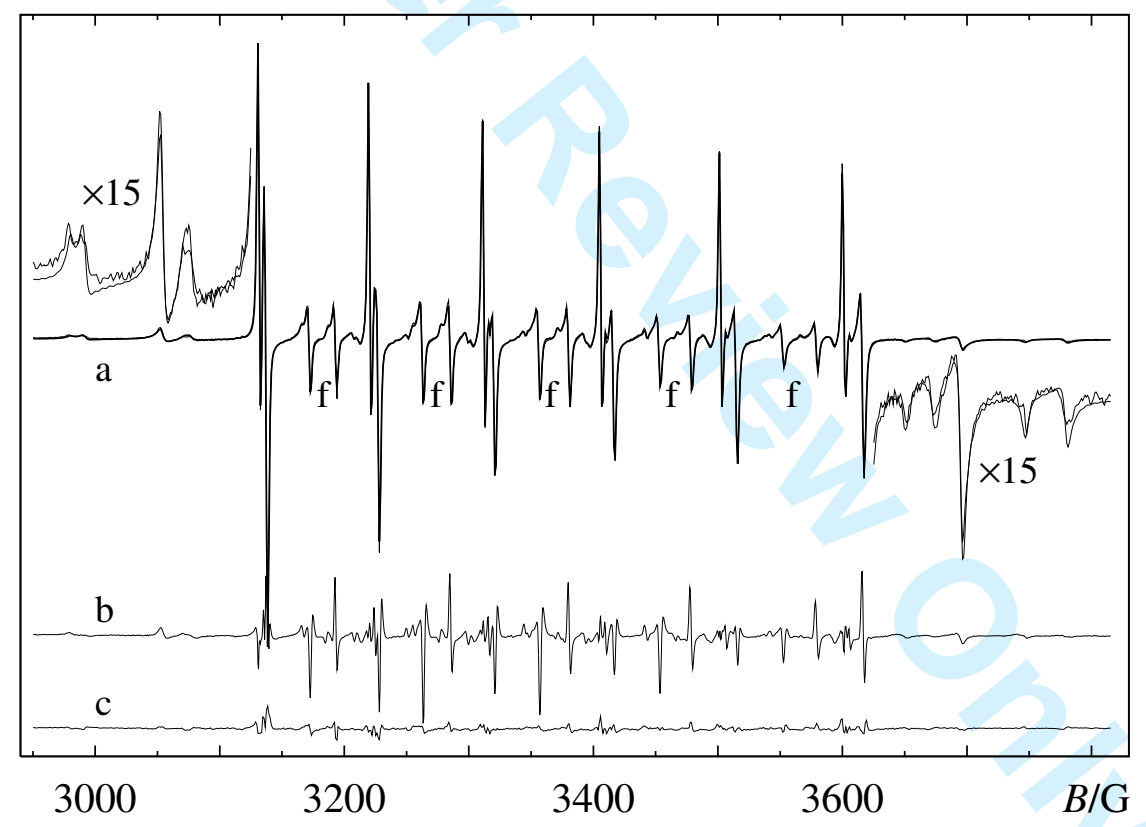


Figure 2: Experimental and calculated room-temperature S-band spectrum of $\mathrm{CaCO}_{3}: \mathrm{Mn}^{2+}$. The experimental spectrum (noisy trace) was obtained at room temperature with $\nu=3.8660 \mathrm{GHz}$.

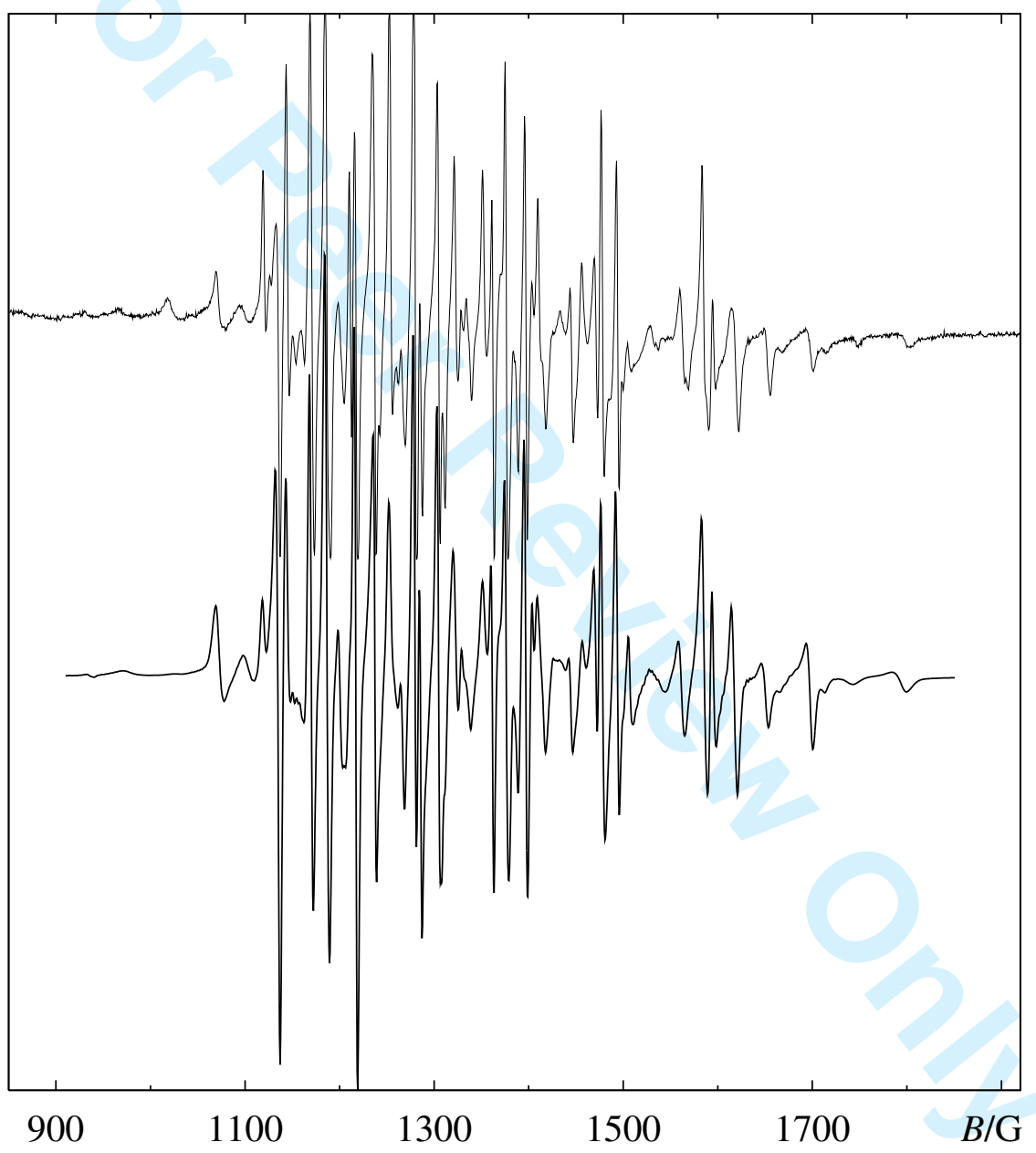

URL: http://mc.manuscriptcentral.com/tandf/tmph 
Figure 3: Experimental and calculated room-temperature L-band spectrum of $\mathrm{CaCO}_{3}: \mathrm{Mn}^{2+}$. The experimental spectrum (noisy trace) was obtained at room temperature with $\nu=1.1078 \mathrm{GHz}$.

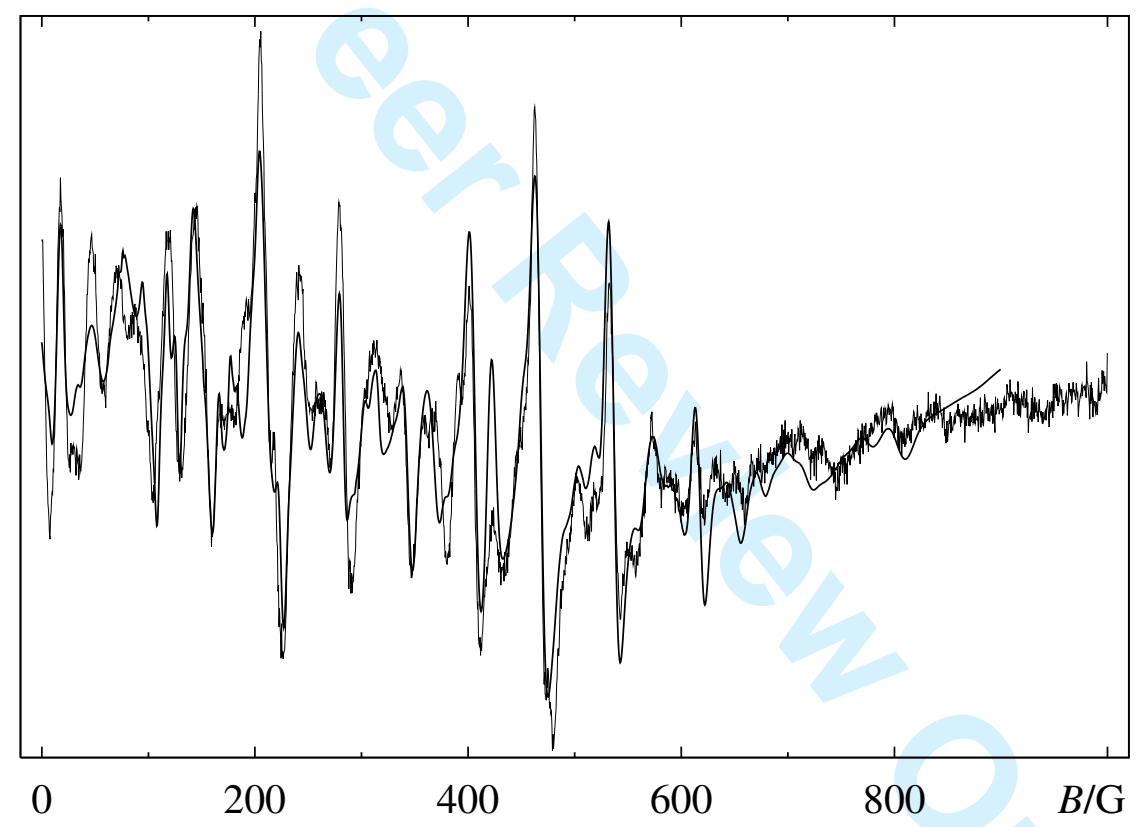


Figure 4: Energy level diagram obtained with $D<0, A_{\|}, A_{\perp}<0$, and $B \| z$. The arrow in the bottom of the figure indicates the energy equivalent of the microwave quantum $\nu=1.1078 \mathrm{GHz}$.

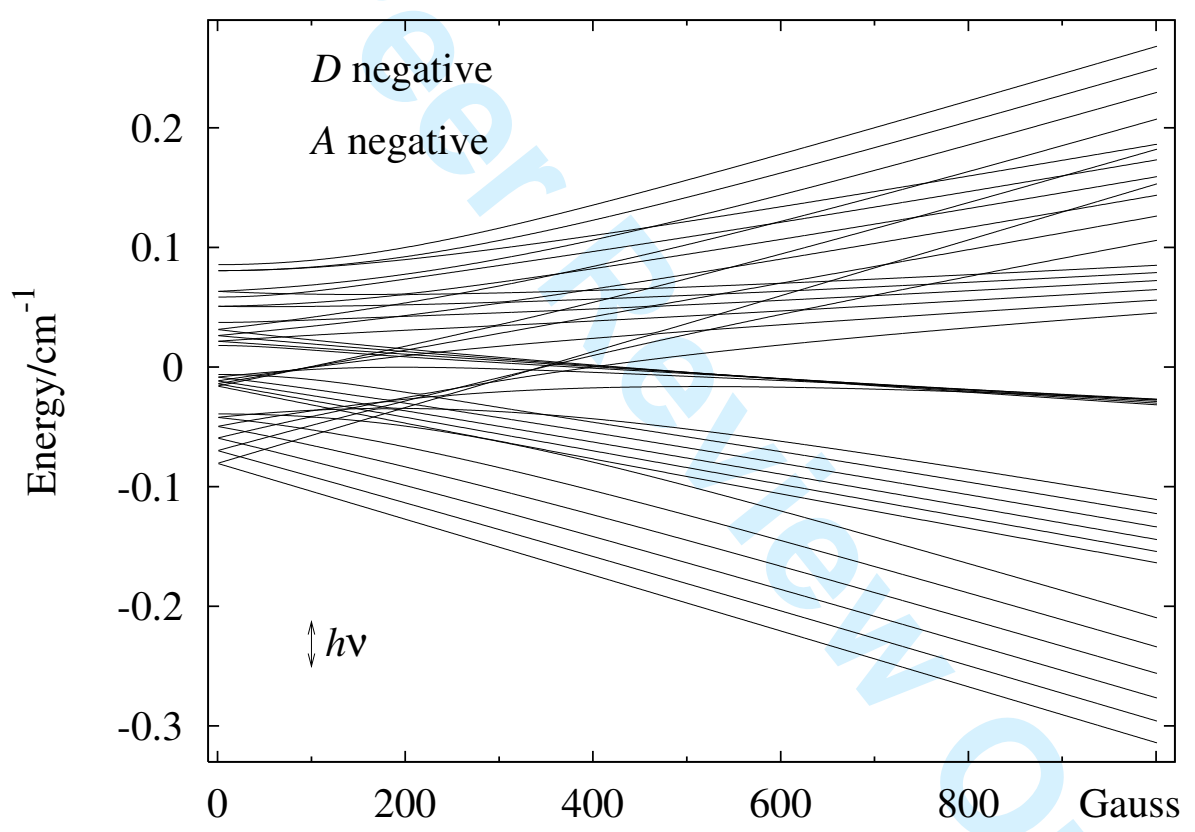


Figure 5: Energy level diagram obtained with $D<0, A_{\|}, A_{\perp}<0$, and $B \| z$. The arrow in the bottom of the figure indicates the energy equivalent of the microwave quantum for $\nu=1.1078 \mathrm{GHz}$.

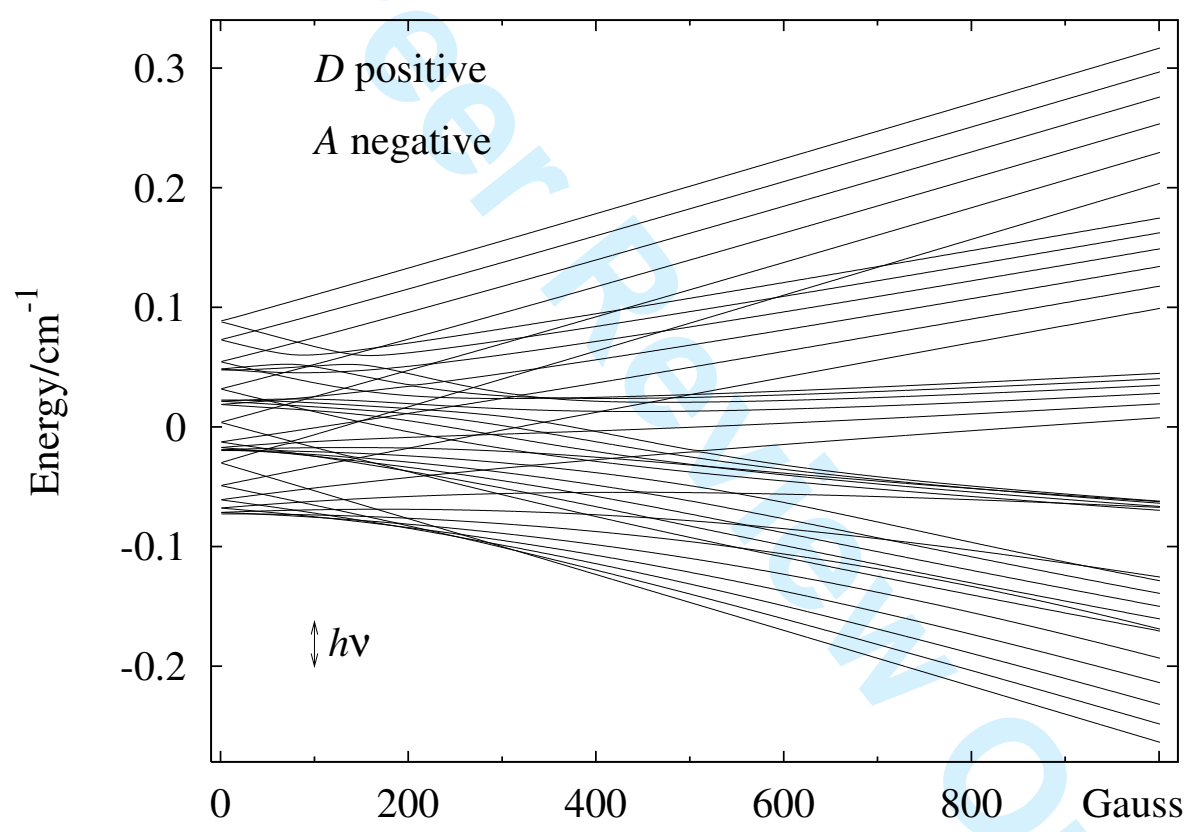


Figure 6: Single-crystal 3.002 $\mathrm{GHz}$ room-temperature spectra of $\mathrm{Mn}^{2+}$ impurities in calcite in the field range 100-1750 Gauss. Upper trace: Experimental spectrum obtained by using a loop-gap resonator and the following experimental conditions: time constant 1 second, conversion time 3 seconds, modulation amplitude 1 Gauss, and a field resolution of 0.14 Gauss. No lines were detected above 1750 Gauss. Lower trace: Computed spectrum using the parameters given in the text. The angle between the unique crystallographic axis and the magnetic field was approximately 12 degrees.

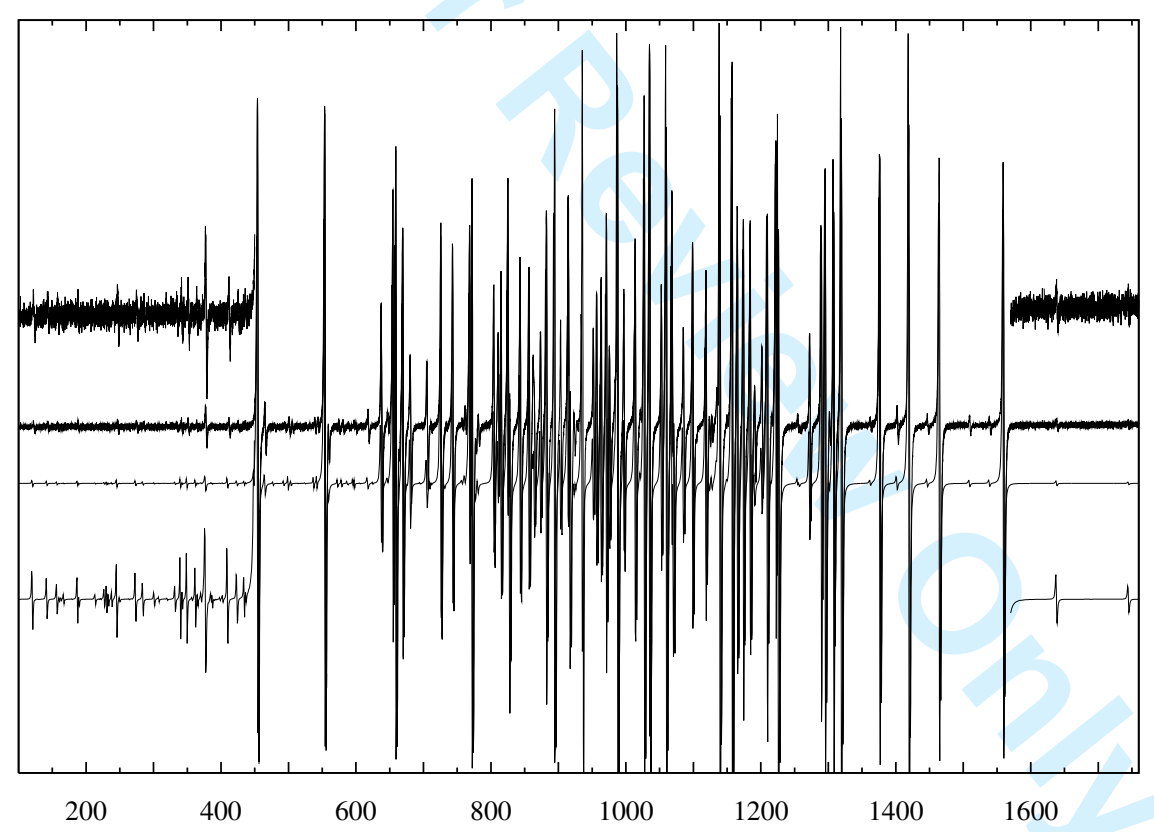




\section{A Computations}

The parameters of eqn (1) were extracted from the experimental spectra by standard $\chi^{2}$ minimization

$\chi^{2}=\sum_{i} \frac{\left[y_{\exp , i}\left(B_{i}\right)-y_{\text {calc }, i}\left(B_{i} ; \boldsymbol{q}\right)\right]^{2}}{\sigma_{i}^{2}}$

making use of the Leuvenberg-Marquardt algorithm [17]. $y_{\exp , i}$ and $y_{\text {calc }, i}$ is the experimental and calculated intensity, respectively, obtained for the $i$-th field value $B_{i} . \boldsymbol{q}$ designates the parameters to be extracted from the spectrum. $\sigma_{i}$ is the experimental uncertainty of the $i$-th experimental intensity. In our computations, this factor is composed of two terms

$\sigma_{i}^{2}=\sigma_{n}^{2}+\left(\frac{\partial y_{i}}{\partial B}\right)^{2} \sigma_{B}^{2}$

where $\sigma_{n}$ is the noise level common to all field points and $\sigma_{B}$ is the field uncertainty taken to be constant $(0.1 \mathrm{G})$ in the field interval.

At each field value the contribution to the derivative spectrum from any pair of energy levels $a, b$ is computed as the field derivative of

$y=\left(P_{a b} p_{a}-P_{b a} p_{b}\right) \times f\left(h \nu, \Delta_{a b}, \Gamma\right)$.

Here, $P_{a b}$ and $P_{b a}$ is the transition probability for the upward and downward transition, respectively. $p_{a}$ and $p_{b}$ is the thermal population of level $a$ and $b$, respectively. $f()$ is a bandshape function with the energy equivalent of the microwave radiation, the energy separation between levels $a$ and $b$, and a bandwidth as arguments, respectively.

In order to introduce some flexibility into the bandshape we use as bandshape function a popular approximation to the Voigt profile [20], namely a linear compination of a Lorentzian $L$ and a Gaussian $G$ lineshape with common bandwidth and centre

$$
f=a L+(1-a) G
$$


Finally, to account for the different bandwidth of the numerous lines in the spectra we model the bandwidth for the transition involving energy levels $a$ and $b$ as

$w_{a b}^{2}=w_{x}^{2} l_{x}^{2}+w_{y}^{2} l_{y}^{2}+w_{z}^{2} l_{z}^{2}+\sum_{i}\left(\frac{\partial \Delta_{a b}}{\partial q}\right)^{2} \sigma_{q_{i}}^{2} \quad$,

where $w_{x}, w_{y}$, and $w_{z}$ are common residual bandwidths for all energy level pairs, and $l_{x}, l_{y}$, and $l_{z}$ are direction cosines of the magnetic field vector with respect to the molecular coordinate system. The terms in the summation account for that the parameters do not have sharp values but rather may be distributed around a mean value. The form eqn (7) assumes that this parameter distribution is gaussian with a width of $\sigma_{q_{i}}$. We are well aware of that eqn (7) is only correct bandwidth to use if the bandshape itself as well as parameter distribution are both gaussian. For a bandshape described by eqn (6), eqn (7) should be modified. However, such a modification would not alter the qualitative outcome of using eqn (7), namely that transitions involving energy levels being more or less sensitive to the parameter $q_{i}$ will become broad and narrow, respectively. 


\section{References}

[1] Hurd, F. K.; Sachs, M.; Hershberger, W. D. Phys. Rev. 1954, 93, 373383

[2] Abragam, A.; Bleaney, B. Electron Paramagnetic Resonance of Transition Ions. Oxford University Press, Oxford 1970

[3] Matarese, L. M. J. Chem. Phys. 1961, 34, 336. Kikuchi, C.; Matarese, L. M. J. Chem. Phys. 1960, 33, 601-606

[4] Hodges, J.A.; Serway, R. A. J. Chem. Phys. 1968, 49, 2857-2858

[5] Mankowitz, J.; Low W. Phys. Rev. 1970, 2, 28-32

[6] Golding, R. M.; Tennant, W. C. Mol. Phys. 1974, 28, 167-175

[7] Tennant, W. C. J. Magn. Reson. 1974, 14, 152-159

[8] Barberis, G. E; Calvo, R.; Maldonado, H.G. Zarate, C.E. Phys. Rev. B 1975, 12, 853-860

[9] Eidels-Dubovoi, S.; Beltrán-Lopez, V. J. Magn. Reson. 1978, 32, 441449

[10] Beltrán-Lopez, V.; Castro-Tello, J. J. Magn. Reson. 1980, 39, 437-460

[11] Beltrán-Lopez, V.; Jiménez, J. J. Magn. Reson. 1982, 48, 302-308

[12] Shepherd, R. A.; Graham, W. R. M. J. Chem. Phys. 1984, 81, 60806084

[13] Armiento, G.; Attanasio, D.; Platania, R. Archaeometry 1997, 39, 309 319 
[14] Duliu, O. G.; Dinescu, L. C.; Skliros, D. J. Trace Microprobe Tech. 1999, 17, 165-175

[15] Attanasio, D.; Platania, R. J. Magn. Reson. 2000, 144, 322-329

[16] Garribba, E.; Micera, Giovanni. Magn. Res. Chem. 2006, 44, 11-19

[17] Preuss, W. H.; Flannery, B. P.; Teukolsky, S. A.; Vetterling, W. T. Numerical Recipes in $C$ : The art of scientific computing Cambridge University Press 1988

[18] Mabbs, F E.; Collison, D. Electron Paramagnetic Resonance of d Transition Metal Compounds, Elsevier 1992

[19] Klitgaard, S. K.; Galsbøl, F; Weihe, H. Spectrochimica Acta 2006, A63, $836-839$

[20] Bruce, S. D.; Higinbotham, J.; Marshall, I.; Beswich, P. H. J. Magn. Reson. 2000, 142, 57-63 
Motecular Physics

\section{$D$ negative}

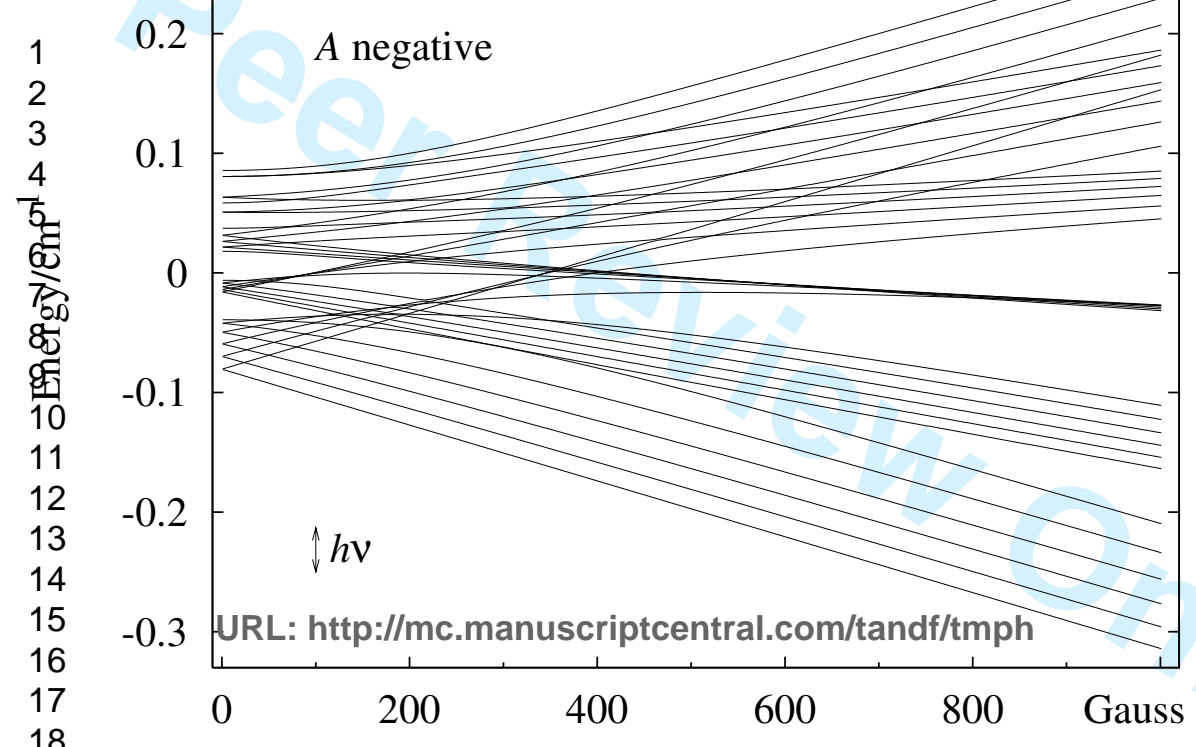




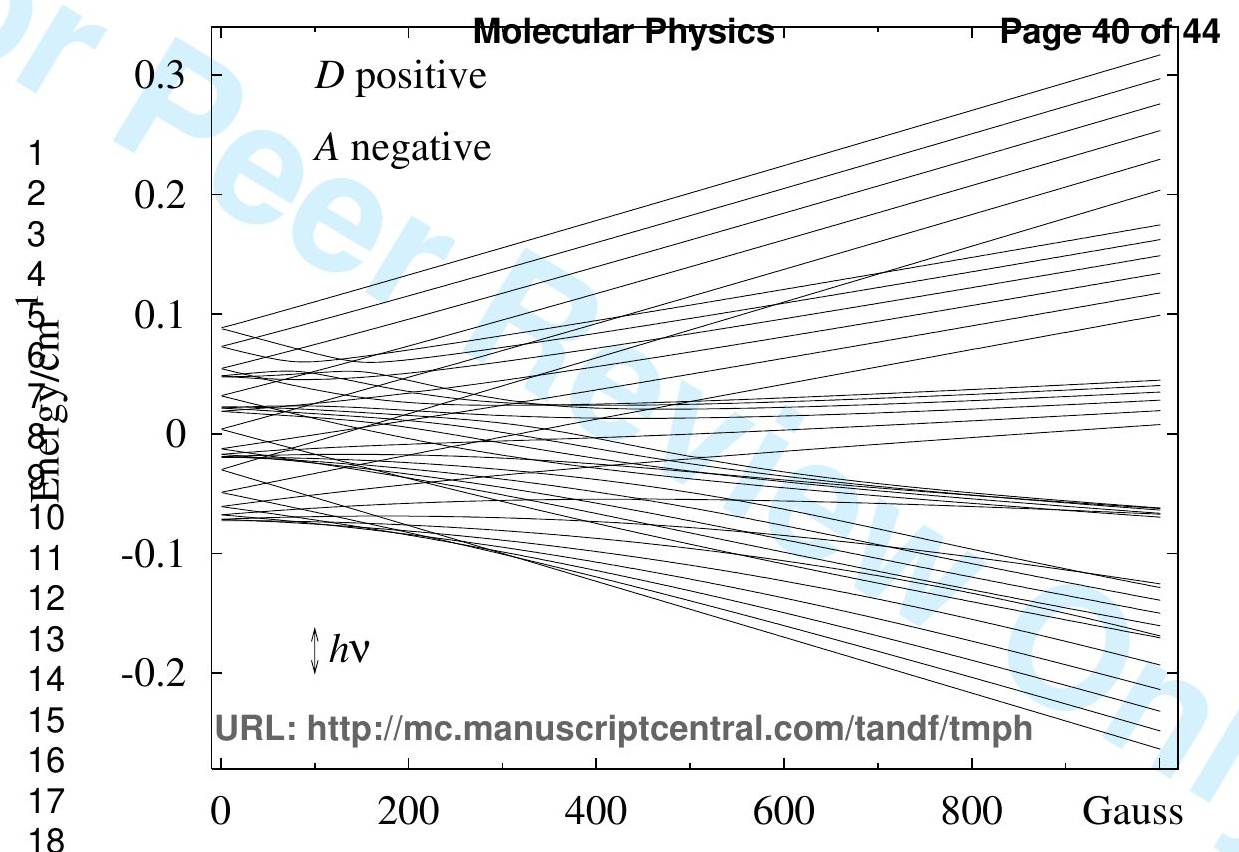




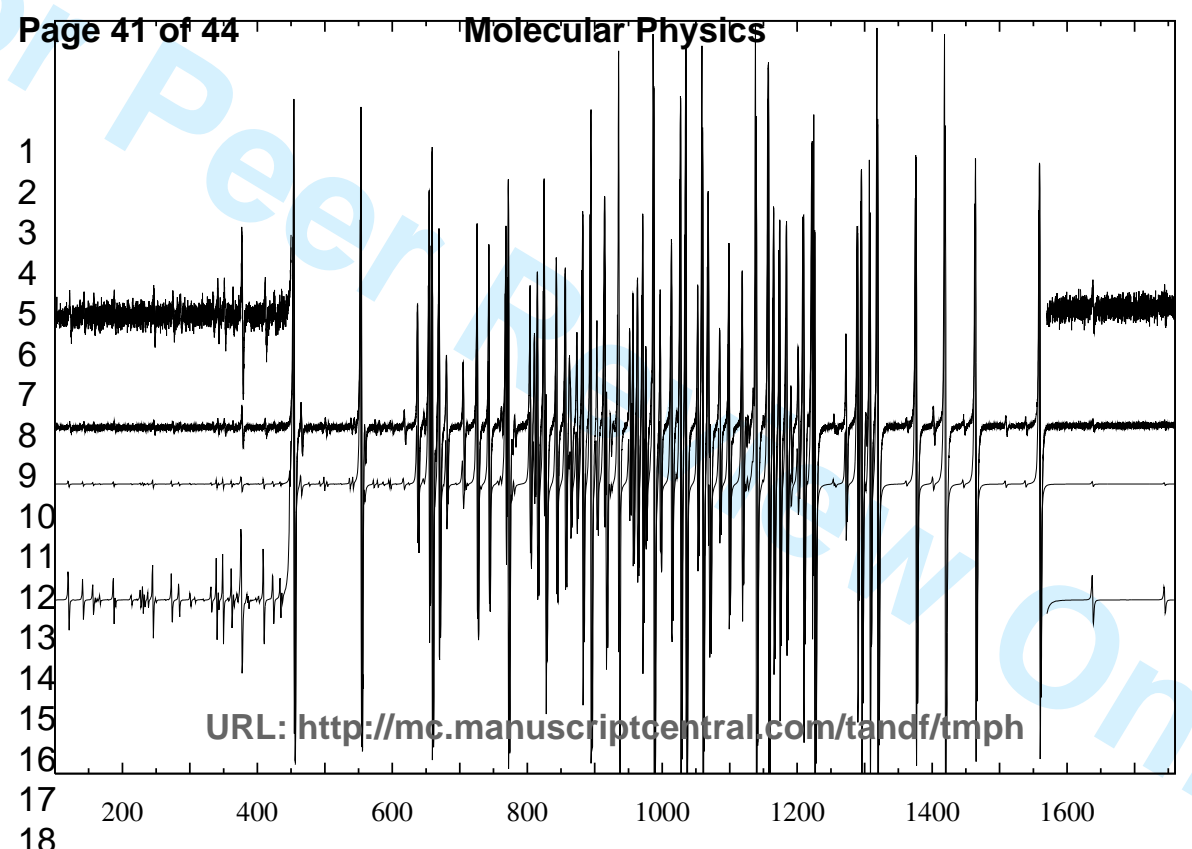




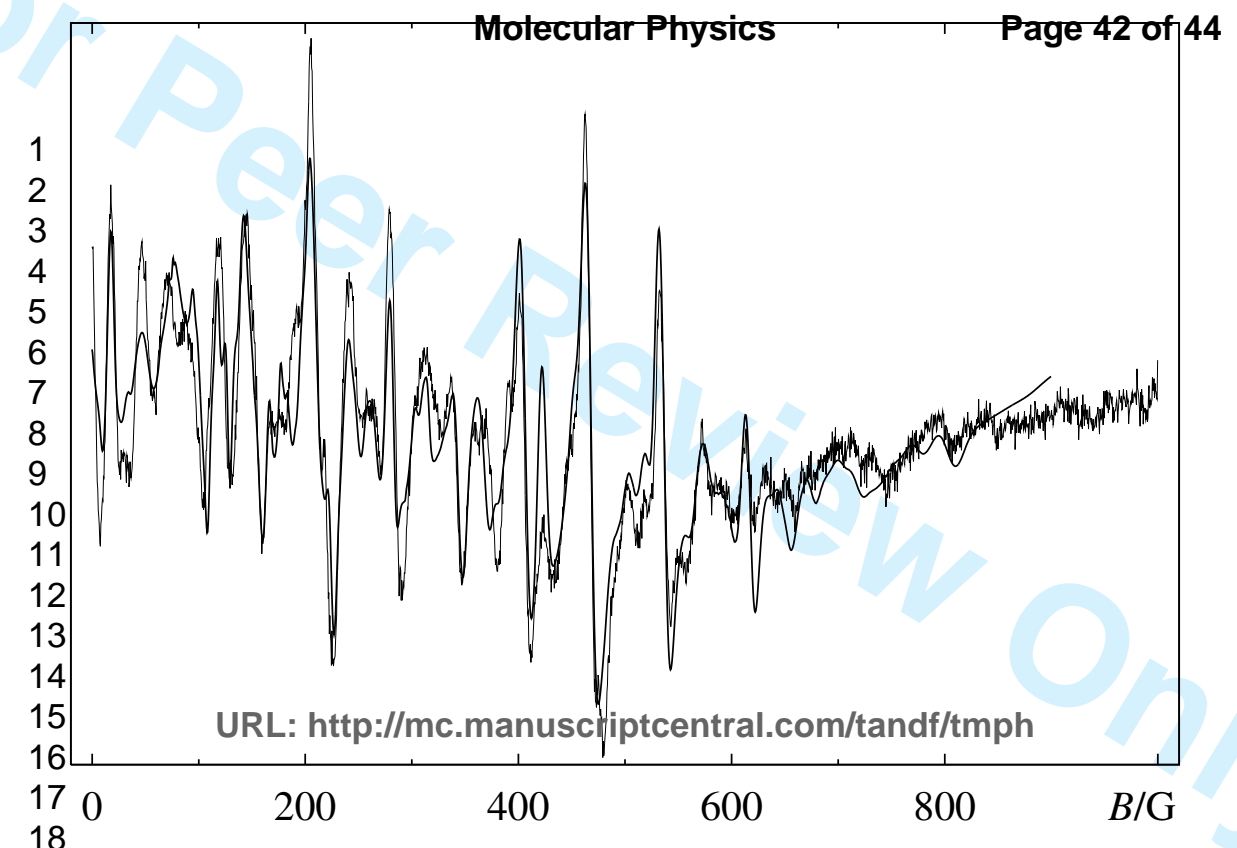




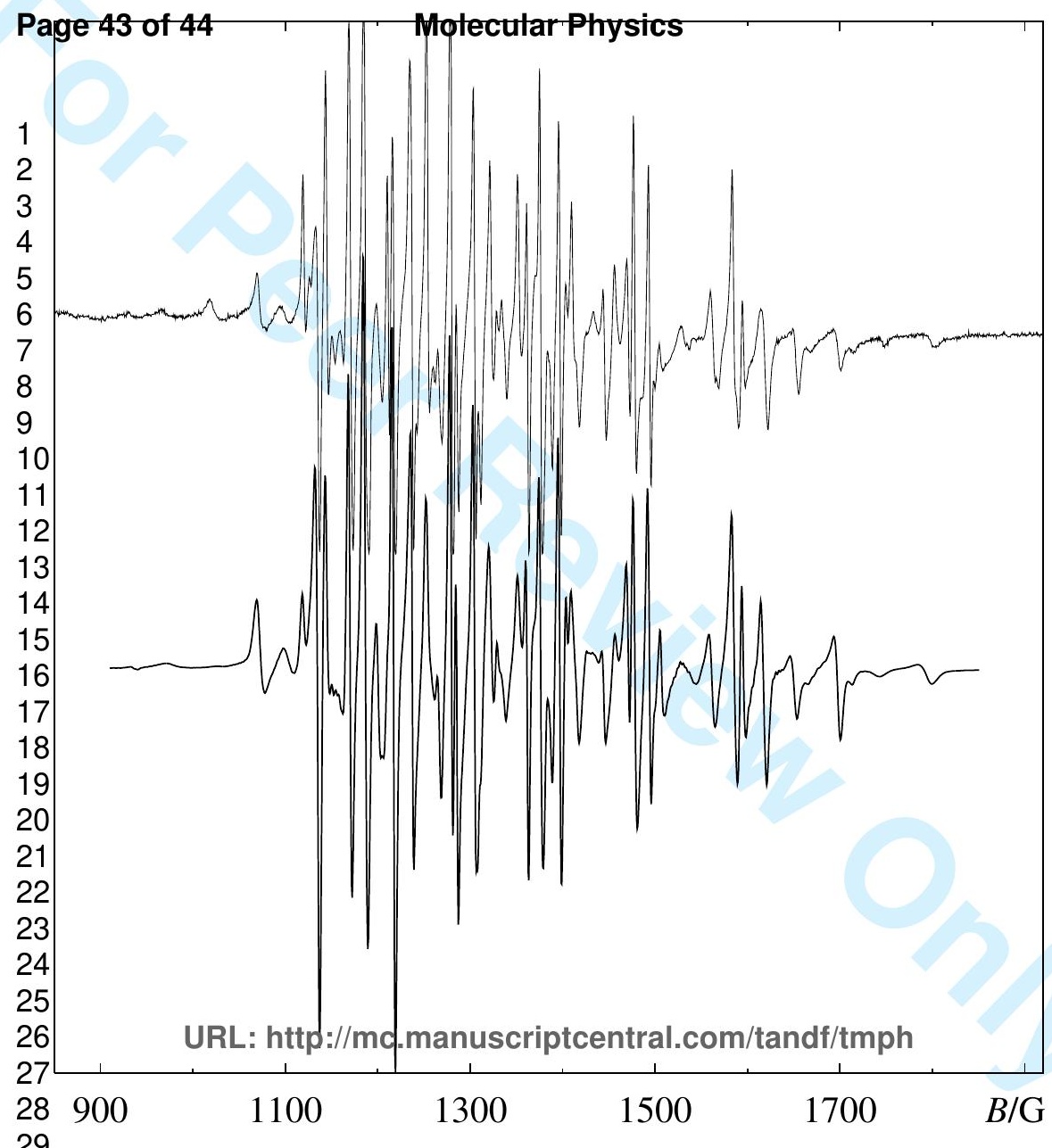


